\title{
IN VITRO ANTIBACTERIAL PROPERTIES OF EL-870, A NEW SEMI-SYNTHETIC MACROLIDE ANTIBIOTIC ${ }^{\dagger}$
}

\author{
E. E. OSE \\ Eli Lilly and Company, \\ Box 708, Greenfield, IN 46140, U.S.A. \\ (Received for publication August 16, 1986)
}

\begin{abstract}
A new macrolide antibiotic, EL-870, 20-deoxo-20-(3,5-dimethylpiperidin-1-yl)desmycosin, has been prepared by chemical modification of desmycosin. In vitro, against selected animal bacterial pathogens, it inhibited growth of Pasteurella multocida, Pasteurella haemolytica, Mycoplasma hyopneumoniae, Actinobacillus pleuropneumoniae, Streptococcus suis, Actinomyces pyogenes and certain other bacteria at levels of $6.25 \mu \mathrm{g} / \mathrm{ml}$ or less. In general, the MICs for Gram-negative enteric bacteria have been $>50 \mu \mathrm{g} / \mathrm{ml}$. Concentrations equivalent to $4 \times$ the MIC value were bactericidal for Pasteurella sp. EL-870 had other antibacterial properties which were characteristic of macrolide antibiotics.
\end{abstract}

EL-870 was selected from a large number of derivatives of desmycosin obtained by chemical modification of the $\mathrm{C}-20$ aldehyde ${ }^{2)}$ for further evaluation to determine its potential for therapeutic use in animals. Selection was based upon in vitro antibacterial activity, the prevention of mortality when tested by parenteral and oral administration in day-old chicks challenged with Pasteurella multocida and other criteria related to chemical syntheses. EL-870 is one of a small group of desmycosin derivatives with in vitro activity equivalent to desmycosin but with activity superior to desmycosin when administered orally for the treatment of pasteurellosis in chicks.

The in vitro antibacterial activity of EL-870 against selected animal bacterial pathogens and certain other antibacterial properties of the compound are reported in this paper. Special emphasis was given to the determination of the sensitivity of Pasteurella sp. to EL-870 due to the importance of these bacteria as pathogens in respiratory diseases of animals.

\section{Materials and Methods}

\section{EL-870}

The chemical structure of EL-870, 20-deoxo-20-(3,5-dimethylpiperidin-1-y1)desmycosin, is shown in Fig. 1. Desmycosin is readily produced from tylosin by mild acid hydrolysis to remove the terminal sugar mycarose. EL-870 is prepared from desmycosin by reductive amination of the C-20 aldehyde with a mixture of cis and trans-3,5-dimethylpiperidine.

EL-870 used for the in vitro studies in this paper, except for the compounds used in the comparison of the antibacterial activity of the cis and the trans isomers, was $75 \sim 80 \%$ pure and consisted of approximately $85 \%$ cis to $15 \%$ trans. The trans isomer is an approximate $1: 1$ mixture of diastereomers. Chromatography on silica gel, [elution with $\mathrm{MeOH}-\mathrm{CH}_{2} \mathrm{Cl}_{2}-\mathrm{NH}_{4} \mathrm{OH}(49.5: 49.5: 1)$ ], furnished fractions which were predominantly cis $(>95: 5)$ or trans $(16: 84)$ for the side-by-side comparative study. EL-870 was used as the free base in these studies.

Bacterial Isolates

Eighty-five of the Pasteurella sp. isolates tested had been isolated from the respiratory tract of cattle at veterinary diagnostic laboratories located in Texas, Oklahoma or Arizona during a period

\footnotetext{
Presented previously in part: See ref 1 .
} 
Fig. 1. 20-Deoxo-20-(3,5-dimethylpiperidin-1-yl)desmycosin, EL-870.

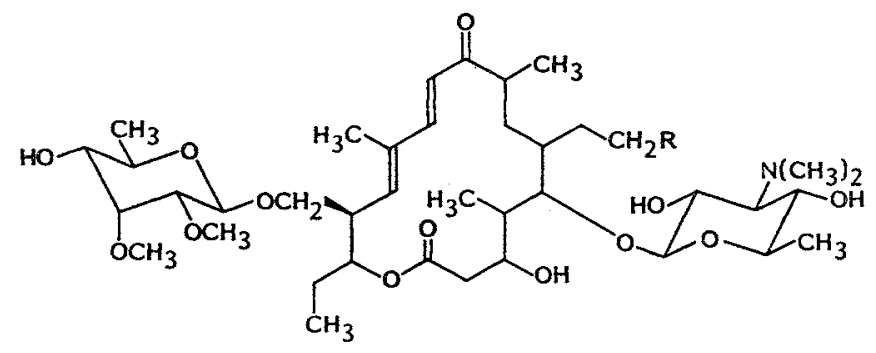

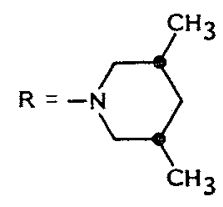

cis $(85$ 용)

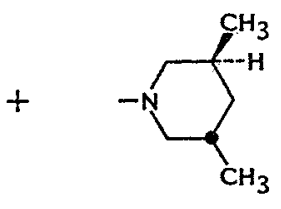

trans $(15 \%)$

from November, 1984 to March, 1985. Ninety-five of the Pasteurella haemolytica isolates were bovine nasal isolates obtained from three groups of cattle shipped from Kentucky to the College of Veterinary Medicine, Purdue University, in 1983, 1984 and 1985. Most of these 95 were resistant to more than one antibacterial agent. This resistance was reported to be plasmid derived and included penicillin, tetracycline, ampicillin, streptomycin and sulfonamides ${ }^{32}$. Six of the porcine $P$. multocida isolates were toxigenic CARTER ${ }^{4)}$ capsular serotype $\mathrm{D}$, while the remainder were CARTER serotype $A$ isolates. All other bacterial isolates had been collected from infected animals over a period of several years.

\section{Antibacterial Activity and Properties}

Most MIC values were determined by standard microtiter or tube broth-dilution tests using Mueller-Hinton broth (Gibco Labs.). The agar dilution test procedure was used with a few of the bacteria. With certain of the bacteria, appropriate enrichment media were required. Cultures of the isolates were diluted, dependent upon the species, so that the inoculum contained $10^{3}$ to $10^{5}$ colony forming units (cfu) per well. For most of the bacteria, incubation was at $37^{\circ} \mathrm{C}$ for $18 \sim 20$ hours. Similar test procedures were used with selected bacteria to compare the antibacterial activity of the cis and trans isomers of EL-870 and to test erythromycin and tylosin-resistant isolates for sensitivity to EL-870.

\section{Effect of Certain Factors on the MIC}

To determine the effect of EL-870 concentration on possible in vitro bactericidal activity of EL870 , three isolates of $P$. multocida and three of $P$. haemolytica were used to inoculate Mueller-Hinton broth containing EL-870 at concentrations equal to $1 \times, 2 \times$ and $4 \times$ the MIC value vs. each of the bacteria. Initial bacterial concentration was $10^{5} \mathrm{cfu} / \mathrm{ml}$. Samples were collected after incubation at $37^{\circ} \mathrm{C}$ for $0,2,4$ and 20 hours, and ten-fold dilutions were plated using duplicate Tryptose agar plates. Colonies on these plates were counted after 20 hours' incubation at $37^{\circ} \mathrm{C}$.

In vitro development of bacterial resistance to EL-870 was measured using isolates of $P$. multocida, $P$. haemolytica or Actinomyces pyogenes. These organisms were passaged daily for 15 days in MuellerHinton broth which contained two-fold dilutions of EL-870. Inoculum for each successive test was taken from the highest concentration of EL-870 in which growth had occurred in the preceding test. The MIC was recorded daily.

The effect of $\mathrm{pH}$ on the antibacterial activity of EL-870 was appraised by determination of the MIC for isolates of Pasteurella sp. and A. pyogenes in Mueller-Hinton media with the $\mathrm{pH}$ adjusted to 7.0 or 8.0. Various inoculum dilutions of the same bacterial cultures were tested using Mueller-Hinton 
Table 1. In vitro antibacterial activity of EL-870 (excluding Pasteurella sp.).

\begin{tabular}{|c|c|c|c|}
\hline Microorganisms & $\begin{array}{c}\mathrm{MIC} \\
(\mu \mathrm{g} / \mathrm{ml})^{\mathrm{a}}\end{array}$ & Microorganisms & $\begin{array}{c}\mathrm{MIC} \\
(\mu \mathrm{g} / \mathrm{ml})^{a}\end{array}$ \\
\hline Staphylococcus aureus & 0.78 & Actinobacillus pleuropneumoniae & 1.56 \\
\hline Streptococcus agalactiae & 3.12 & Bordetella bronchiseptica & 12.50 \\
\hline S. suis & $3.12^{b}$ & Mycoplasma hyopneumoniae & 0.78 \\
\hline Erysipelothrix rhusiopathiae & 0.195 & M. hyorhinis & 12.50 \\
\hline Actinomyces pyogenes & $0.024^{b}$ & M. gallisepticum & $0.048^{b}$ \\
\hline Clostridium perfringens & 3.12 & M. dispar & $0.097^{\circ}$ \\
\hline C. sordellii & 3.12 & M. alkalescens & 0.048 \\
\hline Fusobacterium necrophorum & 3.12 & M. bovirhinis & 0.024 \\
\hline Treponema hyodysenteriae & $>50.00$ & M. bovoculi & 0.048 \\
\hline Escherichia coli & 50.00 & Acholeplasma laidlawii & 0.024 \\
\hline Salmonella typhimurium & $>50.00$ & A. modicum & 0.048 \\
\hline S. choleraesuis & $>50.00$ & & \\
\hline
\end{tabular}

a MIC reported is the value that was found most often for each bacterium.

b Clinical isolates with an MIC $\geq 50 \mu \mathrm{g} / \mathrm{ml}$ have been found.

Table 2. Cumulative in vitro antibacterial activity of EL-870 against Pasteurella $\mathrm{sp}$.

\begin{tabular}{|c|c|c|c|c|c|c|c|c|}
\hline \multirow{2}{*}{$\begin{array}{l}\text { Microorganism } \\
\text { (No. isolates) }\end{array}$} & \multicolumn{8}{|c|}{ Cumulative number and (percent) isolates inhibited } \\
\hline & 0.39 & 0.78 & 1.56 & 3.12 & 6.25 & 12.5 & 25 & 50 \\
\hline \multicolumn{9}{|l|}{ P.m. (102) } \\
\hline Bovine (55) & $2(3.6)$ & $3(5.4)$ & $12(21.8)$ & $30(54.5)$ & $50(90.1)$ & $54(98.2)$ & $55(100)$ & - \\
\hline Porcine $\left(36^{*}\right)$ & - & $1(2.8)$ & $8(22.2)$ & $20(55.6)$ & $36(100)$ & - & 一. & - \\
\hline Avian (11) & - & - & - & $7(63.6)$ & $11(100)$ & - & 一 & 一 \\
\hline \multicolumn{9}{|l|}{ P.h. (155) } \\
\hline Bovine only & - & $17(11.0)$ & $127(81.9)$ & $149(96.1)$ & $153(98.7)$ & $154(99.3)$ & - & $155(100)$ \\
\hline
\end{tabular}

* Includes 7 CARTER type D toxigenic isolates.

P.m.: Pasteurella multocida. P.h.: Pasteurella haemolytica.

broth to determine the effect of inoculum size on the MIC. The influence of serum or plasma on the MIC vs. these same bacteria was also determined. Freshly-collected, filter-sterilized serum or plasma, obtained from individual calves or pigs which had not received antimicrobial treatment, was mixed in equal volumes with Mueller-Hinton medium. MIC values in the media with serum or plasma were compared to the MIC in medium only.

\section{Results}

\section{In Vitro Antibacterial Activity}

The in vitro antibacterial activity of EL-870 (see in Table 1) was primarily against certain Gramnegative and Gram-positive bacteria, anaerobes and Mycoplasma sp. The majority of the Gramnegative enteric bacteria were not sensitive to EL-870. The activity against Actinobacillus pleuropneumoniae was somewhat surprising, since these bacteria are not usually sensitive to macrolide antibiotics. All $29 \mathrm{~A}$. pleuropneumoniae isolates tested were sensitive. As indicated in the table, resistant isolates of certain species normally sensitive to EL-870 have been found.

The $\mathrm{MIC}_{95}$ of EL-870 for $P$. multocida isolates was $6.25 \mu \mathrm{g} / \mathrm{ml}$ (Table 2). Ninety-five $\%$ of the $P$. haemolytica isolates were inhibited by $3.12 \mu \mathrm{g} / \mathrm{ml}$. All of the $P$. haemolytica isolates with known plasmid mediated multiple antibacterial resistance were sensitive to EL-870 at a level of 3.12 $\mu \mathrm{g} / \mathrm{ml}$. There were no effects on the MIC of EL-870 based upon Pasteurella serotype. Sensitivity 
Table 3. In vitro antibacterial activity of the cis and trans isomers of EL-870.

\begin{tabular}{lcc}
\hline \multirow{2}{*}{ Microorganisms } & \multicolumn{2}{c}{$\mathrm{MIC}(\mu \mathrm{g} / \mathrm{ml})$} \\
\cline { 2 - 3 } & \multicolumn{1}{c}{ cis } & trans \\
\hline Staphylococcus aureus & 0.78 & 0.78 \\
Streptococcus agalactiae & 3.12 & 3.12 \\
Mycoplasma gallisepticum & 0.39 & 0.78 \\
M. synoviae & 0.097 & 0.097 \\
M. hyopneumoniae & 0.78 & 0.78 \\
Pasteurella multocida & 3.12 & 6.25 \\
$P$. multocida & 3.12 & 6.25 \\
$P$. multocida & 3.12 & 6.25 \\
$P$. multocida & 1.56 & 3.12 \\
$P$. haemolytica & 1.56 & 3.12 \\
$P$. haemolytica & 3.12 & 3.12 \\
P. haemolytica & 3.12 & 6.75 \\
Bordetella bronchiseptica & 12.50 & 12.50 \\
Escherichia coli & 25.00 & 50.00 \\
\hline
\end{tabular}

to EL-870 of the recent clinical isolates of $P$. haemolytica and $P$. multocida was similar to that of the older isolates. Species origin of $P$. multocida isolates did not appear to affect the tested (Table 3). With the other eight isolates, the MIC for the cis isomer was one dilution lower than that of the trans. The in vitro activity of the isomers was not markedly different.

Selected isolates of Mycoplasma gallisepticum, A. pyogenes and $P$. multocida resistant to tylosin and erythromycin were also resistant to EL-870. Concentrations of EL-870 in the growth medium of $P$. haemolytica at $1 \times$ or $2 \times$ the MIC level resulted in slight reductions in cfu's after 2,4 or 20 hours' incubation (Fig. 2). At $4 \times$ the MIC concentration, EL-870 was bactericidal after 20 hours' incubation. Results were similar with $P$. multocida isolates.

Fifteen consecutive passages of bacteria in broth containing sub-lethal concentrations of EL-870 resulted in a gradual, slight to moderate increase in the MIC. For example, with three isolates of $P$. haemolytica, the mean initial MIC was $1.3 \mu \mathrm{g} / \mathrm{ml}$, while at the 15 th passage, the new MIC was 5.2 . Similar values for three isolates of $A$. pyogenes were 0.012 and 0.024 .

A reduction in $\mathrm{pH}$ of the test medium from 8.0 to 7.0 resulted in a one to eight-fold decrease in the MIC of EL-870 for the 12 bacteria evaluated which is typical for macrolide antibiotics. With Pasteurella sp., an increase in the inoculum of $10^{4} \mathrm{cfu}$ resulted in a zero to four-fold increase in the MIC of EL-870. Similar findings occurred with A. pyogenes. The MIC of EL-870 was not increased or was increased by a factor of only two, when determined in the presence of $50 \%$ bovine or porcine serum or plasma. Serum binding is not marked with EL-870.

\section{Discussion}

EL-870, a new macrolide antibiotic has been found to have an antimicrobial spectrum characteristic of macrolide antibiotics. In addition, EL-870 had significant activity in vitro against $A$. pleuropneumoniae. This activity has been demonstrated in our laboratory in pigs challenged in- 
tranasally with $A$. pleuropneumoniae and treated parenterally with EL-870. It is especially active against isolates of $P$. multocida and $P$. haemolytica. Only $2 / 257$ Pasteurella isolates tested had MICs of $\geq 25 \mu \mathrm{g} / \mathrm{ml}$. These results are indicative of the potential for use of EL-870 as a treatment of pasteurellosis in animals.

The finding that a level of EL-870 equivalent to $4 \times$ the MIC is bactericidal for Pasteurella sp. after 20 hours' incubation, suggests that this compound could exhibit bactericidal in vivo activity against these bacteria. The possibility of this occurring would be enhanced if EL-870 is found to concentrate in lung tissue in a fashion similar to certain other macrolide antibiotics ${ }^{5)}$. Studies in progress, which will be described in later publications, demonstrate the effectiveness of EL-870 as a treatment in cattle with naturally occurring pasteurellosis. Development of EL-870 for the treatment of respiratory disease in animals appears feasible, but will be dependent upon the results of target animal efficacy tests, toxicological testing, tissue residue studies and other factors.

\section{References}

1) OSE, E. E.: In vitro antibacterial properties of EL-870. Program and Abstracts of the 25th Intersci. Conf. on Antimicrob. Agents Chemother., No. 1146, p. 302, Minneapolis, Sept. $29 \sim$ Oct. 2, 1985

2) Debono, M.; K. E. Willard, H. A. Kirst, G. D. Crouse \& E. E. Ose: Synthesis and structure-activity studies of 20-deoxo-20-substituted amino-macrolide antibiotics. Program and Abstracts of the 25th Intersci, Conf. on Antimicrob. Agents Chemother., No. 1145, p. 302, Minneapolis, Sept. 29 Oct. 2, 1985

3) Boyce, J. R. \& R. L. MortaR: Plasmid profile analysis of bovine isolates of Pasteurella haemolytica. Am. J. Vet. Res. 47: 1204 1206, 1986

4) CARTER, G. R.: Studies on Pasteurella multocida. A hemagglutination test for the identification of serological types. Am. J. Vet. Res. 16: 481 484, 1956

5) Thomson, T. D.: Tylosin distribution in normal and pneumonic swine. Proc. Am. Assoc. Swine Pract. 1984: 59 64, 1984 